Ophthalmologe 2011 · 108:5-6

DOI 10.1007/s00347-010-2254-0

Online publiziert: 24 . Dezember 2010

(c) Springer-Verlag 2010

\author{
U. Pleyer \\ Charité, Universitäts-Augenklinik Campus Virchow Klinikum, Berlin
}

\title{
Biologika in der Augenheilkunde
}

spektrum für Biologika rücken wir in dieser Ausgabe von Der Ophthalmologe unsere Patienten mit entzündlichen Augenerkrankungen in den Mittelpunkt.

Fokussiert gehen wir dabei in beiden Heften auf die Zytokininhibitoren ein. Sie bedeuten für die Behandlung der rheumatoiden Arthritis und Spondylarthropathien eine neue Behandlungsqualität. Auf die grundlegenden biotechnologischen Voraussetzungen ihrer Entwicklung gehen Oliver Frey und Thomas Kamradt (Institut für Immunologie, Jena) in ihrem Beitrag ein. Die schrittweise Entwicklung der Biologika führte zu humanisierten Antikörpern und Antikörperfragmenten, die uns als anti-VEGF-Therapie (Ranibizumab, Lucentis) gut bekannt sind. Die Weiterentwicklung antientzündlich wirksamer Biologika ist längst nicht abgeschlossen. Zurzeit sind mehr als 160 monoklonale Antikörper in der klinischen Erprobung; in Phase II/III befinden sich mindestens 13 weitere, die bald in die Klinik eingeführt werden könnten.

Welcher Nutzen ist für unsere Patienten mit entzündlichen Augenerkrankungen zu erwarten? Durch die Behandlungserfolge der Rheumatologen mit TumorNekrose-Faktor (TNF)-Blockern konnte bei Patienten mit begleitender Uveitis ein positiver Effekt auf die Augenbeteiligung nachgewiesen werden.

\section{- Auch bei der Uveitis kommt \\ TNF eine zentrale Rolle zu.}

TNF wurde bei Uveitispatienten in den betroffenen Augen in erhöhter Konzentration nachgewiesen, und es ist an der Gewebezerstörung wesentlich beteiligt $[2,3]$. Es ist daher nicht verwunderlich, dass sich die umfassendsten Erfahrungen zur Anwendung von TNF-Blockern und deren Wirkung auf die intraokulare Entzündung aus rheumatologischen Studien ergaben. Der Beitrag von Pleyer et al. aus dem Berliner Uveitiszentrum fasst daher fachübergreifend die bisherigen Erfahrungen zusammen.

Dabei kann resümiert werden:

- Patienten mit bisher unbefriedigend behandelbaren Erkrankungen (z. B. M. Behçet; sympathischer Ophthalmie, Uveitis bei juveniler, idiopathischer Arthritis) profitierten z. T. erheblich von einer anti-TNF-Blockade.

- Patienten mit Spondylarthropathie wiesen unter anti-TNF-Behandlung signifikant seltener Rezidive ihrer begleitenden Uveitis auf.

- Es ließen sich klare Unterschiede verschiedener Präparate auf die Behandlung entzündlicher Augenerkrankungen feststellen.

- Es liegt bisher allerdings nur eine kontrollierte, randomisierte Studie zur Behandlung der Uveitis vor (die einen klaren Vorteil gegenüber einer Cyclosporin-A-Kontrollgruppe aufwies.

- Alle Behandlungen erfolgen bisher als „Off-label-Anwendungen“.

Erfreulicherweise besteht zunehmend Interesse an der Behandlung von entzündlichen Augenerkrankungen als Indikation für Biologika. Mehrere Zulassungsstudien sind inzwischen initiiert. Neben der bereits bekannten Blockade von TNF und Interleukin (IL)-6 könnten zukünftig auch andere Zytokine wie IL-17 und IL-23 Ziel therapeutischer Ansätze bei entzündlichen Augenerkrankungen sein. Interes-
Retina und Kornea zur Verfügung. Als ein weiteres interessantes Indikations- 
santerweise haben einige hoffnungsvolle Kandidaten hochgesteckte Erwartungen nicht erfüllt und sind heute eher therapeutischen „Nischen“ vorbehalten [1]. Für viele Experten kam der hohe Nutzen der TNF-Blockade eher unerwartet, während andere Zielstukturen diesem Zytokin eher übergeordnet zu sein schienen. So enttäuschte die Blockade des proinflammatorischen Zytokins IL-1, erwies sich allerdings als „durchschlagend effektiv“ beim eher seltenen autoinflammatorischen $\mathrm{Mu}$ ckle-Wells-Syndrom [1]. Einer Reihe von Neuentwicklungen blieb die kommerzielle Marktreife gänzlich verwehrt.

Überraschungen wiesen Biologika anfangs auch bei den unerwünschten Wirkungen auf. Dies zeigte sich bei Einführung der Therapie - und noch fehlender klinischer Erfahrung - in einer deutlichen Rate reaktivierter Infektionen mit M. tuberculosis. Heute ist eine Tbc-Abklärung vor anti-TNF-Therapie bei allen Patienten obligat. Auf die sehr wichtige Nachbeobachtung und das Monitoring bei intraokularer Entzündung geht Talin Barisani aus der Wiener Universitätsklinik ein. Es gilt, bei diesen hochpotenten (kostenintensiven) Biologika

- Wirksamkeit (Nutzen/Benefit),

- Verträglichkeit (Toxizität) und

- Therapierisiko (schwere unerwünschte Nebenwirkungen v. a. im Langzeitverlauf)

möglichst zuverlässig einzuschätzen. Der Beitrag aus der Wiener Klinik weist dabei auf wichtige praktikable Kontrollparameter hin. Gleichzeitig zeigt er, welch große Lücken sich beim Monitoring unserer Patienten noch weiterhin auftun.

Wir können erwarten, dass wir erst am Anfang einer Entwicklung vielfältiger, neuer Therapieoptionen stehen. Wesentliche Impulse sind auch weiterhin aus aktiven Nachbardisziplinen zu erwarten. Dies betrifft z. B. die Blockade der Neovaskularisation, die wesentlich von Erkenntnissen der Onkologie und Diabetologie profitierte, sowie der Entzündungshemmung durch Innovationen aus Rheumatologie und Transplantationsmedizin.

Die hohen Erwartungen, die Paul Ehrlich an seine "Zauberkugeln“ vor über 100 Jahren stellte, gehen somit weitgehend in Erfüllung ...

\section{Korrespondenzadresse}

Prof. Dr. U. Pleyer

Charité, Universitäts-Augenklinik

Campus Virchow Klinikum

Augustenburger Platz 1, 13353 Berlin

uwe.pleyer@charite.de

\section{Literatur}

1. Alexander T, Klotz O, Feist E et al (2005) Successful treatment of acute visual loss in Muckle-Wells syndrome with interleukin 1 receptor antagonist. Ann Rheum Dis 64:1245-1246

2. Santos Lacomba M, Marcos Martin C, Gallardo Galera JM et al (2001) Aqueous humor and serum tu mor necrosis factor-alpha in clinical uveitis. Ophthalmic Res 33:251-255

3. Torun N, Callizo J, Orlic N et al (2005) Serum cytokine receptor levels in noninfectious uveitis. Ophthalmic Res 37:112-116

\section{Ausschreibung \\ der Forschungsförderung 2011 durch die Sarkoidose Stiftung}

Die gemeinnützige Sarkoidose Stiftung schreibt für 2011 die finanzielle Förderung von Forschungsvorhaben zur Sarkoidose und/oder andere granulomatöser Erkrankungen aus.

Es sollen Forschungsvorhaben gefördert werden, die sich der Grundlagenforschung oder der klinisch orientierten Erforschung dieser Krankheitsbilder widmen. Es können Personal-, Sach- und Reisekosten bis zu einer Gesamtsumme von 10.000,- Euro gefördert werden, die in der Regel innerhalb eines Jahres verausgabt werden sollten.

\section{Der Antrag besteht aus:}

- einer auch für Fachfremde verständlichen Projektskizze auf nicht mehr als 10 Seiten,

- einer einseitigen Zusammenfassung zum Thema,

- dem Lebenslauf,

- dem Verzeichnis bisheriger Veröffentlichungen,

- ggf. bereits abgewickelte Forschungsprojekte.

Dem Antrag können zusätzliche Unter lagen zur näheren Darlegung des Forschungsvorhabens beigefügt werden.

Die Bewerber werden gebeten, ihren Antrag bis zum 31.03.2011 (Poststempel) in zweifacher Ausführung per Post oder als Wordbzw. PDF-Datei per E-Mail bei der Sarkoidose Stiftung einzureichen:

Sarkoidose Stiftung

Geschäftstelle

Uerdinger Str. 43

D-40668 Meerbusch

E-Mail: forschung@sarkoidose-stiftung.de 